\title{
Des interrogations éthiques qui laissent perplexe
}

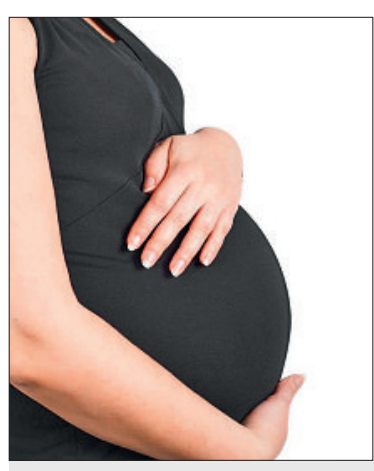

Césarienne de convenance? Césarienne sous contrainte? Des deux côtés, des enjeux éthiques non négligeables...

1 Martin J. Biomédecine et procréation. Revue médicale suisse. 2005; 1:453-8.

2 Les césariennes de la discorde. 24 Heures (Lausanne), 21 décembre 2010. p. 33.

3 Martin J. Le patient peut-il exiger des prestations du médecin? Bull Méd Suisses. 2010, 91(38):1504.

4 Minkoff H, Drapkin Lyerly A. Samantha Burton and the rights of pregnant women. Hastings Center Report. Nov./Dec. 2010; 40. No. 6. p. 13-5.

5 Kolder VE, Gallagher J, Parsons MT. Court-ordered obstetrical interventions. New England Journal of Medicine. 1987; 316:1192-6.

6 Appel JM. Medical kidnapping: rogue obstetricians vs. pregnant women. Huffington Post. January 24; 2010.

7 Je profite ici de relever question qui m'est parfois posée par des étudiants ou des confrères - que, en ce qui concerne l'interruption de grossesse, il n'y a aucune situation où, dans nos régimes juridiques en tout cas, on pourrait obliger une femme à avoir une interruption.
En Suisse, une grossesse sur trois se termine aujourd'hui par une césarienne. Au moment de mes études, on était au-dessous de $10 \%$. Les temps changent, la médecine progresse et veut mettre toutes les chances du côté des patient-e-s, en vue de la meilleure qualité de vie possible et d'un inconfort minimal. Toutefois, le fait est qu'on se demande jusqu'où ira à l'avenir l'impact des «variantes technicisées de la manière de faire des enfants dont nous avions l'habitude» [1].

Les organisations de sages-femmes s'émeuvent de cette désaffection de l'accouchement par voie basse - je me souviens avoir vu en Angleterre des affiches de cliniques promouvant la césarienne sous le slogan «Saving the love channel». Récemment j'ai été frappé d'apprendre que, dans un service universitaire alémanique, on accepte de routine de faire une césarienne sur le souhait de la femme quand, après avoir reçu les explications adéquates sur la nonnécessité de l'opération, la femme persiste dans sa demande. Extraits d'un interview dans 24 Heures: «Avant de tomber enceinte, je me suis assurée qu'il m'était permis d'accoucher par césarienne. Il était exclu que je mette un enfant au monde par voie basse.» Certains considéreraient l'accouchement vaginal comme un «bizutage» (ce genre d'épreuves initiatiques entre étudiants ou membres d'une corporation) [2]! Well... j'avoue me sentir vraiment ici d'une ancienne génération. Pourtant, je me suis beaucoup engagé pour la reconnaissance et l'inclusion dans la loi des droits des patients; mais la question semble de plus en plus actuelle de savoir si les patients sont en droit d'exiger des prestations de leur médecin [3].

La même interrogation, césarienne ou pas, peut se poser en sens inverse; elle est discutée dans le dernier numéro de la revue de bioéthique Hastings Center Report [4]. C'est de savoir s'il est admissible, et quand, qu'un tribunal ordonne la césarienne d'une femme contre sa volonté. Je m'y suis intéressé comme médecin officiel (cantonal), en rapport avec le fait que, dans certains Etats des USA, une césarienne d'autorité avait pu être décrétée chez des femmes toxicomanes proches du terme dont on juge qu'elles mettent gravement en danger le bien-être de l'enfant [5]. Néanmoins, de notre côté de l'Atlantique, aucun pays n'admet que, si la personne concernée le refuse, une aussi sérieuse atteinte à l'intégrité physique puisse se justifier, même au nom du bien de l'enfant qu'elle porte.
Ici une remarque: Kolder et al. montrent que les femmes qu'on a contraintes à une césarienne contre leur gré tendaient à être pauvres, issues d'une minorité et souvent ne parlaient pas anglais. Un exemple de plus de cette constante de santé publique que les soins prodigués aux gens dépendent de leur statut et que les disparités socio-économiques se traduisent en injustices (NB: cela peut s'appliquer à la césarienne de convenance, très vraisemblablement plus aisément accordée à la femme de classe moyenne ou aisée).

Minkoff et Dryerly décrivent des mouvements de balancier aux Etats-Unis en ce qui concerne les césariennes «forcées», citant plusieurs recours acceptés par les tribunaux supérieurs qui ont jugé qu'il n'était pas justifié de les pratiquer. Préoccupation: «Bien que les obstétriciens acceptent le droit des femmes de refuser des gestes médicaux, les vingt dernières années ont vu une érosion de ces droits. Cet affaiblissement est pour une part lié à deux choses: les guerres sans fin à propos de l'avortement et la vision que la relation entre femme et fotus a fondamentalement un caractère de conflit.» Avec ce commentaire: «Aujourd'hui les contraintes sur les droits des femmes enceintes sont souvent plus prononcées que les limites posées aux droits des parents sur leurs enfants déjà nés.» Cela rappelle une idée qui venait devant certains débats, à l'époque, sur le régime du délai; on se demandait si ceux qui refusaient absolument l'interruption de grossesse étaient prêts à consacrer autant d'efforts et d'argent à prendre soin de manière adéquate des enfants nés dans des circonstances très défavorables.

Pour conclure, d'un autre auteur américain ce conseil aux femmes: «Posez à votre gynécologue la question suivante: peut-il y avoir une circonstance dans laquelle vous refuserez de me laisser décider moi-même de ce qui concerne mon traitement?» [6]. Intéressant: avec sans doute tous ceux qui se préoccupent d'éthique, j'estime que le droit de la femme de refuser est entier et déterminant, au cas où une instance quelconque s'avisait de lui imposer une césarienne [7]; je ne suis pas à l'aise s'agissant de son droit, par convenance, de requérir une césarienne. Convient-il que je sois plus attentif à mes propres tendances paternalistes ou machistes?

Jean Martin, membre de la Commission nationale d'éthique et de la rédaction du BMS 